\title{
ON LATTICE-POINTS IN A RANDOM SPHERE
}

BY K. CHANDRASEKHARAN AND RAGHAVAN NARASIMHAN

Communicated by A. A. Albert, September 15, 1966

1. I. M. Vinogradov and A. G. Postnikov, in their one-hour report on Recent developments in analytic number theory at the International Congress of Mathematicians (Moscow, 1966) have referred to a recent result of A. A. Judin on the lattice-point problem for a random circle. If $(\alpha, \beta)$ is an arbitrary point in the plane, and $A(x ; \alpha, \beta)$ denotes the number of lattice-points inside and on the circumference of a circle with $(\alpha, \beta)$ as centre and $x^{1 / 2}$ as radius, then Judin's result, as stated in the above-mentioned report, is that

$$
\limsup _{x \rightarrow \infty} \frac{|A(x ; \alpha, \beta)-\pi x|}{x^{1 / 4}}>c>0,
$$

and the proof, according to the report, is by the application of arguments from the theory of almost periodic functions. This is of interest in view of the known result [3] that

$$
A(x ; \alpha, \beta)-\pi x=O\left(x^{1 / 4+\epsilon}\right), \quad \epsilon>0,
$$

for almost all points $(\alpha, \beta)$.

It is our object to show that the following result, hence also Judin's, is a direct consequence of a theorem of ours on the average order of arithmetical functions:

$$
\begin{gathered}
\limsup _{x \rightarrow \infty} \frac{A(x ; \alpha, \beta)-\pi x}{x^{1 / 4}}>0, \\
\liminf _{x \rightarrow \infty} \frac{A(x ; \alpha, \beta)-\pi x}{x^{1 / 4}}<0 .
\end{gathered}
$$

This result is true not only in the plane, but in $k$ dimensions, for $k \geqq 2$. Instead of $A(x ; \alpha, \beta)$, one can also consider its higher averages of order $\rho \geqq 0$, the proof being the same.

2. Theorem. If $\left(\alpha_{1}, \cdots, \alpha_{k}\right)$ is an arbitrary point in $k$-space, $k \geqq 2$, and $A\left(x ; \alpha_{1}, \cdots, \alpha_{k}\right)$ denotes the number of lattice-points inside and on a sphere with centre $\left(\alpha_{1}, \cdots, \alpha_{k}\right)$, and radius $x^{1 / 2}$, then

$$
\left[A\left(x ; \alpha_{1}, \cdots, \alpha_{k}\right)-\pi^{k / 2} x^{k / 2} / \Gamma(k / 2+1)\right]=\Omega_{ \pm}\left(x^{(k-1) / 4}\right),
$$

as $x \rightarrow \infty$. 
Proof. (i) Let $\alpha_{1}, \cdots, \alpha_{k}$ be given real numbers, not all being integers at the same time. Let $\left(n_{k}\right)$ denote integers. Let $\left(\lambda_{r}\right)$ be the sequence of real numbers $\left\{\left(n_{1}-\alpha_{1}\right)^{2}+\cdots+\left(n_{k}-\alpha_{k}\right)^{2}\right\}$ arranged in increasing order of magnitude. Define

$$
a_{r}=\sum_{\left(n_{1}-\alpha_{1}\right)^{2}+\cdots+\left(n_{k}-\alpha_{k}\right)^{2}=\lambda_{r}} 1 .
$$

Consider the Dirichlet series

$$
\begin{aligned}
\phi(s) & =\sum_{r=1}^{\infty} \frac{a_{r}}{\lambda_{r}^{s}}, \quad s=\sigma+i t, \\
& =\sum_{n_{r}=-\infty}^{\infty} \sum \frac{1}{\left\{\left(n_{1}-\alpha_{1}\right)^{2}+\cdots+\left(n_{k}-\alpha_{k}\right)^{2}\right\}^{*}} .
\end{aligned}
$$

This converges absolutely for $\sigma>k / 2$, and satisfies a functional equation given by

$$
\pi^{-s} \Gamma(s) \phi(s)=\pi^{s-k / 2} \Gamma\left(\frac{k}{2}-s\right) \psi\left(\frac{k}{2}-s\right),
$$

where $\psi$ is represented by the Dirichlet series

$$
\begin{aligned}
\psi(s) & =\sum_{r=1}^{\infty} \frac{b_{r}}{r^{\circ}} \\
& =\sum_{n_{r}=-\infty} \sum_{\left(n_{1}, \cdots, n_{k}\right) \neq(0,0, \cdots, 0)} \frac{\exp \left(2 \pi i\left(n_{1} \alpha_{1}+\cdots+n_{k} \alpha_{k}\right)\right)}{\left(n_{1}^{2}+\cdots+n_{k}^{2}\right)^{*}},
\end{aligned}
$$

where

$$
b_{r}=\sum_{n_{1}+\cdots+n_{k=r}} \exp \left(2 \pi i\left(n_{1} \alpha_{1}+\cdots+n_{k} \alpha_{k}\right)\right) .
$$

Equation (2.1) can be proved directly in the same way as the functional equation of Riemann's zeta-function. If

$$
\theta(\alpha, y)=\sum \underset{n_{r}=-\infty}{\infty} \sum \exp \left(-\left[\left(n_{1}-\alpha_{1}\right)^{2}+\cdots+\left(n_{k}-\alpha_{k}\right)^{2}\right] \pi y\right),
$$

for $\operatorname{Re} y>0$, then

where

$$
\theta(\alpha, y)=y^{-k / 2} \theta_{1}(\alpha, 1 / y)
$$

$$
\begin{aligned}
& \theta_{1}(\alpha, y) \\
& =\sum \underset{n_{r}=-\infty}{\infty} \sum \exp \left(2 \pi i\left(n_{1} \alpha_{1}+\cdots+n_{k} \alpha_{k}\right)-\pi\left(n_{1}^{2}+\cdots+n_{k}^{2}\right) y\right) .
\end{aligned}
$$


If we denote

$$
\theta_{2}(\alpha, y)=\theta_{1}(\alpha, y)-1,
$$

then, for $\sigma>k / 2$, we have

$$
\begin{aligned}
\pi^{-s} \Gamma(s) \phi(s)= & \int_{1}^{\infty} y^{s-1} \theta(\alpha, y) d y \\
& +\int_{1}^{\infty} y^{k / 2-s-1} \theta_{2}(\alpha, y) d y+\frac{1}{s-\frac{1}{2} k},
\end{aligned}
$$

and

$$
\begin{aligned}
\pi^{-s} \Gamma(s) \psi(s)= & \int_{1}^{\infty} y^{s-1} \theta_{2}(\alpha, y) d y \\
& +\int_{1}^{\infty} y^{k / 2-s-1} \theta(\alpha, y) d y-\frac{1}{s} .
\end{aligned}
$$

These two relations show that $\phi(s)$ and $\psi(s)$ are meromorphic functions in the whole $s$-plane, with $\phi$ having a simple pole at $s=k / 2$ with residue $\pi^{k / 2} / \Gamma(k / 2)$. Further $\phi$ and $\psi$ satisfy equation (2.1). Not all the coefficients $\left(b_{n}\right)$ are zero. Hence Theorem 3.2 of [2] is applicable, with $\rho=0, Q_{0}(x)=\pi^{k / 2} x^{k / 2} / \Gamma(k / 2+1)$, and $\theta=(k-1) / 4$, giving what we want.

(ii) If $\alpha_{1}, \cdots, \alpha_{k}$ are all integers, then $\phi(s)=\psi(s)$, and we have Epstein's zeta-function, which is known to satisfy (2.1). The result is again obvious.

REMARK 1. If one starts with a positive-definite quadratic form $Q$ in $k$-variables, with real coefficients, where $k \geqq 2$, one considers the corresponding function

and obtains the result

$$
A(x ; Q, \alpha)=\sum_{Q(n-\alpha) \leq x} 1
$$

$$
A(x ; Q, \alpha)-\pi^{k / 2} x^{k / 2} / \Gamma(k / 2+1)|Q|^{1 / 2}=\Omega_{ \pm}\left(x^{(k-1) / 4}\right),
$$

as $x \rightarrow \infty$, where $|Q|$ is the determinant of $Q$.

REMARK 2. The function $A\left(x ; \alpha_{1}, \cdots, \alpha_{k}\right)$ is integrable and multiperiodic in the $\alpha$ 's, with period 1, and its Fourier expansion is given by

$$
\begin{aligned}
& A\left(x ; \alpha_{1}, \cdots, \alpha_{k}\right) \sim c_{1} x^{k / 2}+c_{2} x^{k / 4} \sum \cdots \sum \\
& \quad \frac{\exp \left(2 \pi i\left(\alpha_{1} n_{1}+\cdots+\alpha_{k} n_{k}\right)\right) J_{k / 2}\left(2 \pi x^{1 / 2}\left(n_{1}^{2}+\cdots+n_{k}^{2}\right)^{1 / 2}\right)}{\left(n_{1}^{2}+\cdots+n_{k}^{2}\right)^{k / 4}} .
\end{aligned}
$$


If one integrates the series on the right, with respect to $x, \rho$ times, where $\rho$ is a sufficiently large integer, one obtains an absolutely convergent series, which is the Fourier series of $A_{\rho}\left(x ; \alpha_{1}, \cdots, \alpha_{k}\right)$, the $\rho$ th integral, with respect to $x$, of $A\left(x ; \alpha_{1}, \cdots, \alpha_{k}\right)$, and is therefore equal to it. Thus one obtains an identity of the form

$$
\begin{aligned}
& \frac{1}{\Gamma(\rho)} \int_{0}^{x} A\left(t ; \alpha_{1}, \cdots, \alpha_{k}\right)(x-t)^{\rho-1} d t \\
= & c_{3} x^{k / 2+\rho}+c_{4} x^{k / 4+\rho / 2} \sum \cdots \sum \frac{J_{k / 2+\rho}\left(2 \pi x^{1 / 2}\left(n_{1}^{2}+\cdots+n_{k}^{2}\right)^{1 / 2}\right)}{\left(n_{1}^{2}+\cdots+n_{k}^{2}\right)^{k / 4+\rho / 2}} \\
& \cdot \exp \left(2 \pi i\left(\alpha_{1} n_{1}+\cdots+\alpha_{k} n_{k}\right)\right) .
\end{aligned}
$$

It is known that this is equivalent to a functional equation of the form (2.1). (See Lemma 5 of [1].)

\section{REFERENCES}

1. K. Chandrasekharan and Raghavan Narasimhan, Hecke's functional equation and arithmetical identities, Ann. of Math. 74 (1961), 1-23.

2. - Functional equations with multiple gamma factors and the average order of arithmetical functions, Ann. of Math. 76 (1962), 93-136.

3. D. G. Kendall, On the number of lattice points inside a random oval, Quart J. Math. Oxford 19 (1948), 1-26.

FORSCHUNGSINSTITUT FÜR MATHEMATIK, E. T. H. ZÜRICH 